\title{
Mindfulness-based Stress Reduction on Unhealthy Emotions and Sleep Quality of Hematopoietic Stem Cell Transplantation Recipients
}

\author{
Wu Xiafen, Huang Guohua*, Wang Chunli, Deng Jinqing \\ Department of Hematology, The First Affiliated Hospital of Jinan University, Guangzhou, China \\ Email address: \\ jndxhgh@163.com (Huang Guohua) \\ ${ }^{*}$ Corresponding author
}

\section{To cite this article:}

Wu Xiafen, Huang Guohua, Wang Chunli, Deng Jinqing. Mindfulness-based Stress Reduction on Unhealthy Emotions and Sleep Quality of Hematopoietic Stem Cell Transplantation Recipients. American Journal of Internal Medicine. Vol. 8, No. 2, 2020, pp. 84-88.

doi: 10.11648/j.ajim.20200802.17

Received: March 9, 2020; Accepted: March 23, 2020; Published: April 17, 2020

\begin{abstract}
Objective: We attempt to explore the effect of mindfulness-based stress reduction on unhealthy emotion and sleep quality of hematopoietic stem cell transplantation recipients. Methods: we enrolled 92 patients who underwent hematopoietic stem cell transplantation recipients in the Hematology Department of our hospital from February 2017 to August 2019 and averagely divided them into control group and observation group according to random number table. Patients in the control group received routine nursing care for hematopoietic stem cell transplantation recipients while patients in the observation group were given mindfulness-based stress reduction for mental intervention based on the routine nursing care. We adopted Self-rating Anxiety Scale (SAS), Self-rating Depression Scale (SDS), and Pittsburgh Sleep Quality Index (PSQI) to assess the patients' emotional state and sleep quality before and after intervention. Results: Before intervention, there was no significant difference in the score on SAS, SDS, and PSQI between two groups $(\mathrm{P}>0.05)$. After intervention, the scores on SAS, SDS, and PSQI of both groups declined obviously and that of the observation group declined much more than that of the control group. There was a significant difference in the score on SAS, SDS, and PSQI between two groups after intervention $(\mathrm{P}<0.05)$. Conclusions: Mindfulness-based stress reduction can relieve unhealthy emotions such as anxiety and depression of hematopoietic stem cell transplantation recipients as well as their mental stress so as to improve their sleep quality.
\end{abstract}

Keywords: Mindfulness-based Stress Reduction, Hematopoietic Stem Cell Transplantation, Unhealthy Emotion, Sleep Quality

\section{Introduction}

Hematopoietic stem cell transplantation (HSCT) is the most effective new treatment for benign and malignant hematological system diseases currently, with a cure rate up to $60 \%-70 \%[1,2]$. The fact that hematopoietic stem cell transplantation requires closed hundred-level laminar air flow ward, and long course of treatment, high cost and high risk bring heavy financial burden and mental stress to the patients and their families. At the same time, nausea, emesis, dental ulcer, stomachache, diarrhea and many other drug toxicities and side effects, as well as thirst for and fear of transplantation cause worry and negative emotions to patients. The unhealthy emotions inevitably affect treatment compliance. Research shows that mental intervention can improve patients' mental stress endurance, and reduce negative emotions [3, 4]. Mental intervention, as an indispensable key part of the nursing process, emphasizes that not only patients' physiological needs should be met, but also their psychological comfort should be improved, so that they can achieve wholeness at physio-psycho-social level during the entire process of diagnosis and treat. Mindfulness-based stress reduction, based on mindfulness meditation, is a method for management of emotion and stress, whose core concept include consciousness, focusing on the present moment and making no comment [5]. It has been widely used to relieve stress and manage emotions by scholars at home and abroad and they have achieved good effects $[6,7]$. The current study employed mindfulness-based 
stress reduction in patients who received hematopoietic stem cell transplantation in the Hematology Department of our hospital from February 2017 to August 2019 and observed this mental intervention's effect on patients' unhealthy emotions and sleep quality. The study is reported as follows.

\section{General Data and Methods}

\subsection{General Data}

We selected 92 patients who underwent hematopoietic stem cell transplantation in the Hematology Department of our hospital from February 2017 to August 2019 as participants. Among them, 20 patients received autologous hematopoietic stem cell transplantation and 72 received allogeneic hematopoietic stem cell transplantation. The inclusion criteria are: (1) patients who received hematopoietic stem cell transplantation and with an age of $\geq 16$; (2) patients with sanity, normal hearing, language expression and understanding; (3) patients whose score on SAS was $\geq 50$ and score on SDS was $\geq 53$ in the screening of unhealthy emotions at the first day entering laminar flow room; (4) patients who had difficulty falling asleep, sleep disruption, early awakening, difficulty going back to sleep after awakening and other sleep problems; (5) patients having signed informed consent. Exclusion criteria are: (1) patients who were under 16; (2) patients who had been diagnosed with insomnia and needed to take sedative hypnotics; (3) those who had past medical history of psychonosema; (4) patients who had severe complications during transplantation and could not participate in the research. We used random number table to averagely divide the 92 patients into observation group and control group. In the observation group, there are 30 males and 16 females, aging from 23 to 57 with an average age of $(47.5 \pm 4.2)$. In the control group, there are 28 males and 18 females, aging from 16 to 61 , with an average age of $(46.8 \pm 4.5)$. There was no significant difference in the sex, age, marital status, occupation, education background, religious belief, payment method of medical expenses and many other general data between two groups $(\mathrm{P}>0.05)$.

\subsection{Methods}

\subsubsection{Control Group}

We employed regular nursing care for hematopoietic stem cell transplantation recipients in the control group including routine care for HSTC, daily mental nursing care, health education related to transplantation and behavior guide. Before entering laminar flow room, nurses conducted overall assessment for the patients. Nurses explained medication, the do's and don'ts, possible adverse reaction, and countermeasures during the four stages of hematopoietic stem cell transplantation, namely preconditioning stage, blood stem cell return infusion stage, bone marrow emptiness stage and hematopoietic stem cell survival stage, to patients in order for elimination of nervousness and fear, and for compliance to treatment. We carefully observed patients' clinical manifestation and emotional change during transplantation.
What's more, we communicated a lot with patients, provided them with psychological counseling, listened to the causes of their negative emotions and answered their doubts to relax them.

\subsubsection{Observation Group}

Based on the routine nursing care as in the control group, we also adopted mindfulness-based stress reduction in the mental intervention for patients in the observation group. Medical staff who have received professional training on mindfulness-based stress reduction gave one-on-one explanation and instruction of the mindfulness-based stress reduction to patients every day. Firstly they told the patients the concept of mindfulness-based stress reduction, and guided them to assume a sitting position or supine position, breathe smoothly and close eyes. At the same time, the medical staff guides the patients to feel the breath and the movement of the abdomen, to just objectively observe instead of making any subjective comment, and told the patients how to deal with negative emotions with "mindfulness". The medical staff also instructed the patients in practicing mindfulness meditation and walking meditation, in focusing on the thoughts and impulsion in their head and feeling how they appeared and disappeared so as to help them accept or at least do not reject and criticize these emotions. The medical staff guided the patients to assume a supine or sitting position and focus on the body, scanning from the toes up to the top of the head, and at the same time, to perceive with heart the feeling of every part of the body. If the patient felt pain or discomfort somewhere, the caregiver could suggest that these pains and discomforts would leave the body with breathing. If the patient grasped the practice of mindfulness-based stress reduction preliminarily, they could practice through the mindfulness-based stress reduction training $C D$ for 2 times a day and 15 minutes each time.

\subsection{Outcome Measurements}

We assessed the two groups on SAS, SDS, general condition questionnaire and PSQI at the first day they entered the laminar flow room and 4 weeks after intervention.

\subsubsection{Self-rating Anxiety Scale (SAS) and Self-rating Depression Scale (SDS)}

The Self-rating Anxiety Scale (SAS) and Self-rating Depression Scale (SDS) are made by William W. K. Zung to evaluate the subjective feelings of individuals with symptoms of anxiety and depression. They are a basis for measuring the degree of anxiety and depression and their changes in treatment [8]. SAS and SDS both include 20 items, mainly for assessment of the frequency of symptoms of each item in the past week. The 4-point scale is used and summation of score of each item is the preliminary total score, which is multiplied by 1.25 and the integer part is kept to get the standard score. A higher standard score indicates more severe depression. According to Chinese norms, the SAS standard score is 50 points, and 50-59 points means mild anxiety, 60-69 points means moderate anxiety, and 70 points and above means 
severe anxiety. The SDS standard score is 53 points, and 53-62 points means mild depression, 63-72 points moderate depression, and 72 points and above severe depression.

\subsubsection{General Condition Questionnaire and Pittsburgh Sleep Quality Index (PSQI)}

The general condition questionnaire includes patient's sex, age, marital state, occupation, education background, religious belief, payment method of medical expenses and other demographic information [9]. PSQI mainly consists of 24 items including 19 self-assessment items and 5 peer assessment items which are composed of 7 factors namely time of falling asleep, sleep efficiency, hour of sleep, sleep disorders, sleep quality, hypnotic and daytime dysfunction. Each factor is scored on a four-point scale of 0-3, and the summation of each factor's score is total PSQI score which ranges from 0-21 points. The higher the total score, the worse the sleep quality. In China, PSQI $>7$ is seen as the reference threshold for adult sleep quality problems.

\subsection{Statistical Analysis}

We used statistical software SPSS23.0 for statistical analysis. We adopted mean \pm standard deviation $(\bar{x} \pm s)$ to present measurement data. $\mathrm{T}$ test for two samples was employed for comparison between two groups and pared-samples $\mathrm{T}$ test for comparison of one group between different times, and $\mathrm{P}<0.05$ means significant difference.

\section{Results}

\subsection{Comparison of Scores of SAS and SDS Between Two Groups Before and After Intervention}

Before intervention, there was no significant difference in the scores of SAS and SDS between two groups $(\mathrm{P}>0.05)$. However, after intervention, the scores in observation groups decreased much more obviously than those in the control group $(\mathrm{P}<0.05)$, as shown in the Table 1 .

Table 1. Comparison of Scores of SAS and SDS between Two Groups before and after Intervention (point, $\overline{x \pm s) .}$

\begin{tabular}{|c|c|c|c|c|c|c|c|c|c|}
\hline \multirow[b]{2}{*}{ Group } & \multirow[b]{2}{*}{$\mathbf{n}$} & \multicolumn{4}{|l|}{ Score of SAS } & \multicolumn{4}{|l|}{ Score of SDS } \\
\hline & & $\begin{array}{l}\text { Before } \\
\text { intervention }\end{array}$ & $\begin{array}{l}\text { After } \\
\text { intervention }\end{array}$ & $t^{*}$ & $p^{*}$ & $\begin{array}{l}\text { Before } \\
\text { intervention }\end{array}$ & $\begin{array}{l}\text { After } \\
\text { intervention }\end{array}$ & $t^{*}$ & $p^{*}$ \\
\hline Observation group & 46 & $57.25 \pm 2.63$ & $34.12 \pm 1.87$ & 12.234 & 0.001 & $58.72 \pm 5.23$ & $38.76 \pm 3.28$ & 12.236 & 0.001 \\
\hline Control group & 46 & $56.87 \pm 2.27$ & $45.23 \pm 2.85$ & 8.168 & 0.001 & $59.25 \pm 5.19$ & $49.75 \pm 4.25$ & 15.827 & 0.001 \\
\hline $\mathrm{t}^{\#}$ & & 0.323 & 3.685 & & & 0.269 & 2.347 & & \\
\hline $\mathrm{p}^{\#}$ & & 0.735 & 0.001 & & & 0.781 & 0.026 & & \\
\hline
\end{tabular}

Note: $t^{*}, p^{*}$ stand for comparison of scores before and after intervention within a group, and $\mathrm{t}^{\#}, \mathrm{p}^{\#}$ stand for comparisons between two groups before and after intervention respectively.

\subsection{Comparison of Scores of Sleep Quality (PSQI) Between Two Groups Before and After Intervention}

Before intervention, there was no significant difference in the score of each factor of sleep except hypnotic and total PSQI score between two groups $(\mathrm{P}>0.05)$. Score of each item and total score in the observation group declined much more than those in the control group with a significant difference $(\mathrm{P}<0.05)$, as shown in Table 2 .

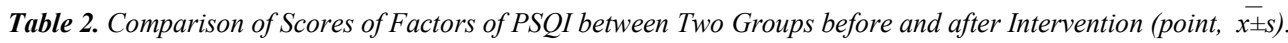

\begin{tabular}{|c|c|c|c|c|c|c|c|c|}
\hline \multirow[b]{2}{*}{ Variables } & \multicolumn{2}{|c|}{ Control group } & \multicolumn{2}{|c|}{ Observation group } & \multirow[b]{2}{*}{ t1/p1 } & \multirow[b]{2}{*}{ t2/p2 } & \multirow[b]{2}{*}{ t3/p3 } & \multirow[b]{2}{*}{ t4/p4 } \\
\hline & $\begin{array}{l}\text { Before } \\
\text { intevention } \\
n=46\end{array}$ & $\begin{array}{l}\text { After } \\
\text { intervention } \\
n=46\end{array}$ & $\begin{array}{l}\text { Before } \\
\text { intervention } \\
n=46\end{array}$ & $\begin{array}{l}\text { After } \\
\text { intervention } \\
n=46\end{array}$ & & & & \\
\hline Sleep quality & $1.58 \pm 0.67$ & $1.47 \pm 0.53$ & $1.56 \pm 0.57$ & $0.81 \pm 0.48$ & $2.879 / 0.002$ & $5.596 / 0.001$ & $0.307 / 0.375$ & $4.415 / 0.001$ \\
\hline Time of falling asleep & $1.63 \pm 0.56$ & $1.58 \pm 0.52$ & $1.64 \pm 0.68$ & $0.98 \pm 0.44$ & $1.827 / 0.036$ & $3.919 / 0.006$ & $0.811 / 0.208$ & $3.564 / 0.001$ \\
\hline Hour of sleep & $1.67 \pm 0.64$ & $1.77 \pm 0.43$ & $1.75 \pm 0.53$ & $1.03 \pm 0.37$ & $1.667 / 0.046$ & $3.151 / 0.001$ & $0.185 / 0.429$ & $1.934 / 0.026$ \\
\hline Sleep efficiency & $1.64 \pm 0.85$ & $1.62 \pm 0.51$ & $1.67 \pm 0.67$ & $0.83 \pm 0.42$ & $2.059 / 0.016$ & $3.247 / 0.001$ & $0.425 / 0.337$ & $2.463 / 0.004$ \\
\hline sleep disorders & $1.91 \pm 0.43$ & $1.64 \pm 0.46$ & $1.85 \pm 0.51$ & $0.78 \pm 0.44$ & $2.094 / 0.017$ & $4.917 / 0.001$ & $0.405 / 0.343$ & $3.145 / 0.001$ \\
\hline hypnotic & $0.19 \pm 0.44$ & $0.15 \pm 0.32$ & $0.14 \pm 0.40$ & $0.09 \pm 0.25$ & $-0.47 / 0.057$ & $-0.663 / 0.714$ & $1.435 / 0.813$ & $1.458 / 0.243$ \\
\hline Daytime dysfunction & $1.78 \pm 0.52$ & $1.68 \pm 0.47$ & $1.78 \pm 0.57$ & $0.75 \pm 0.45$ & $2.406 / 0.007$ & $4.281 / 0.001$ & $0.487 / 0.315$ & $2.686 / 0.003$ \\
\hline Total PSQI score & $10.31 \pm 2.23$ & $9.63 \pm 1.42$ & $10.36 \pm 1.77$ & $5.19 \pm 1.17$ & $2.829 / 0.003$ & $12.682 / 0.001$ & $1.453 / 0.098$ & $7.458 / 0.001$ \\
\hline
\end{tabular}

Note: $\mathrm{t} 1 / \mathrm{p} 1$ stands for comparison of scores before and after intervention in the control group; $\mathrm{t} 2 / \mathrm{p} 2$ stands for comparison of scores before and after intervention in the observation group; $\mathrm{t} 3 / \mathrm{p} 3$ stands for comparison of scores between two groups before intervention; $\mathrm{t} 4 / \mathrm{p} 4$ stands for comparison of scores between two groups after intervention.

\section{Discussion}

Some scholars used Self-rating Anxiety Scale (SAS) to assess the degree of anxiety of hematopoietic stem cell transplantation recipients and found that the occurrence of anxiety during transplantation was up to $100 \%$ [10-11].
Isolation in laminar flow wards from the outside world during transplantation, limited range of activities, changes in life rules, the fact that patients can only communicate with their loved ones through video, while facing medical staff and treatment induced toxicities and adverse reactions, high medical expenses as well as concerns about transplantation failure will inevitably cause a series of psychological stress reactions in patients, 
which will further lead to anxiety, depression and other bad emotions. These negative emotions will affect the development and treatment of patients' diseases. Therefore, we should pay attention to the psychological care for patients in clinical practice, attempting to eliminate the patient's unhealthy emotions to increase their compliance to treatment. Mindfulness-based stress reduction therapy originates from Eastern Zen thought. It is an experience of focusing instantaneous perception objectively and uncritically on the present moment [12]. This study uses mindfulness-based stress reduction to change patients' cognitive behaviors and help patients eliminate or reduce existing bad emotions. After routine nursing intervention and mindfulness-based stress reduction training, comparison of the scores of psychological status of the two groups before and after the intervention shows that the SAS and SDS scores of the two groups are significantly lower than those before the intervention, and the decline in the observation group is more significant $(\mathrm{P}<0.05)$. The results suggest that mindfulness-based stress reduction therapy can improve the mental state of hematopoietic stem cell transplantation recipients and relieve their anxiety, depression and other negative emotions, improving the treatment effect.

Patients undergoing hematopoietic stem cell transplantation have all experienced multiple times of chemotherapy. With the increase in the times of chemotherapy, the patient has more knowledge about chemotherapy, and more of their own disease, and so they are more likely to have anxiety and fear of the disease. In terms of treatment, on the one hand, patients have a strong desire for transplantation, but they are also worried about the failure of the transplantation. At the same time, the transplantation will cost a large amount of medical expenses. These factors cause patients to suffer greater psychological pressure and have a negative impact on their psychology. As a result, patients are in a state of anxiety and restlessness, which leads to reduced sleep quality. On the other hand, the more frequent treatment and care for patients during the transplantation and the noise of the machine in the sterile laminar flow room will inevitably disturb the patient's rest, causing difficulty in falling asleep and frequent waking up. The mindfulness-based stress reduction training, mainly through mindful breathing, meditation, and body scanning, to adjust the patient's bad mood, guide the patient to cultivate an open mind to further relieve their stress, improving their sleep quality [13-15]. In this study, we provide patients with mindfulness-based stress reduction training. The results show that the sleep quality of the two groups is poor before intervention, and there is no significant difference between the two groups $(\mathrm{P}>0.05)$. After intervention, the PSQI scores of the two groups are significantly lower than those before the intervention, and the decline in the observation group is greater with a significant difference $(\mathrm{P}<0.05)$, indicating that mindfulness-based stress reduction training can significantly improve the sleep quality of patients.

\section{Conclusions}

In conclusion, in the current study, employment of mindfulness-based stress reduction in hematopoietic stem cell transplantation recipients can improve their cognitive behavior, relieve their anxiety, depression and other negative emotions and thus increase treatment effect. At the same time, the mindfulness-based stress reduction training, mainly through mindful breathing, meditation, to adjust the patient's bad mood, guide the patient to cultivate an open mind to further relieve their stress. The mindfulness-based stress reduction can relieve mental stress so as to improve sleep quality of hematopoietic stem cell transplantation recipients. However, factors affecting patient's psychological states and sleep quality are various, so comprehensive assessment and study are needed.

\section{References}

[1] Edward A Copelan. (2006). Hematopoietic stem-cell transplantation. N Engl J Med, 354 (17), 1813-1826.

[2] Bertaina, A., \& Roncarolo, M. G. (2019). Graft engineering and adoptive immunotherapy: new approaches to promote immune tolerance after hematopoietic stem cell transplantation. Frontiers in immunology, 10, 1342.

[3] Jiali, L., \& Manlei, W. (2015). Effect of systemic psychological intervention on the emotional state of patients with hematopoietic stem cell transplantation. Journal of Clinical Medicine in Practice, 19 (8): 46-48.

[4] Patel, N. C., Chinen, J., Rosenblatt, H. M., Hanson, I. C., Krance, R. A., \& Paul, M. E., et al. (2009). Outcomes of patients with severe combined immunodeficiency treated with hematopoietic stem cell transplantation with and without preconditioning. Journal of Allergy and Clinical Immunology, 124 (5), 1062-1069. e4.

[5] Math Janssen, Yvonne Heerkens, Wietske Kuijer, Beatrice van der Heijden, \& Josephine Engels. (2018). Effects of mindfulness-based stress reduction on employees' mental health: a systematic review. Plos One, 13 (1), e0191332.

[6] Shaw, J. M., Sekelja, N., Frasca, D., Dhillon, H. M., \& Price, M. A. (2018). Being mindful of mindfulness interventions in cancer: a systematic review of intervention reporting and study methodology. Psycho-Oncology, 27 (4): $1162-1171$

[7] Modi, Alsubaie, Rebecca, Abbott, Barnaby, \& Dunn, et al. (2017). Mechanisms of action in mindfulness-based cognitive therapy (mbct) and mindfulness-based stress reduction (mbsr) in people with physical and/or psychological conditions: a systematic review. Clin Psychol Rev, 55: 74-91.

[8] Mingyuan, Z. Psychiatric Rating Scale Manual, second edition, Changsha: Hunan Science and Technology Press, 1998: 81.

[9] Xiaoyang, D. Manual of Common Psychological Assessment Scales, Beijing: Military Science Publishing House, 2012: 56-155.

[10] Tecchio, C., Bonetto, C., Bertani, M., Cristofalo, D., Lasalvia, A., \& Nichele, I., et al. (2013). Predictors of anxiety and depression in hematopoietic stem cell transplant patients during protective isolation. Psycho-Oncology, 22 (8), 1790-1797. 
[11] El-Jawahri, A. R., Vandusen, H. B., Traeger, L. N., Fishbein, J. N., Keenan, T., \& Gallagher, E. R., et al. (2016). Quality of life and mood predict posttraumatic stress disorder after hematopoietic stem cell transplantation. Cancer, 122 (5), 806-812.

[12] Johansson, B., Bjuhr, H., \& Ronnback, L. (2012). Mindfulness-based stress reduction (mbsr) improves long-term mental fatigue after stroke or traumatic brain injury. Brain Injury, 26 (13-14), 1621-1628.

[13] Andersen, S. R., Würtzen, Hanne, Steding-Jessen, M., Christensen, J., Andersen, K. K., \& Flyger, H., et al. (2013). Effect of mindfulness-based stress reduction on sleep quality: results of a randomized trial among danish breast cancer patients. Acta Oncologica, 52 (2), 336-344.

[14] Xinli, C., Ai, B., Tingting, L., Peichao, Z., \& Iris, C. (2018). Effects of mindfulness-based stress reduction on depression in adolescents and young adults: a systematic review and meta-analysis. Frontiers in Psychology, 9, 1034.

[15] Bohlmeijer, E., Prenger, R., Taal, E., \& Cuijpers, P. (2010). The effects of mindfulness-based stress reduction therapy on mental health of adults with a chronic medical disease: a meta-analysis. Journal of Psychosomatic Research, 68 (6), $0-544$. 